\title{
Comparative Analysis of Radial Forearm Free Flap and Anterolateral Thigh Flap in Tongue Reconstruction after Radical Resection of Tongue Cancer
}

\author{
Yong-Cong Cai ${ }^{a} \quad$ Chao Li $^{a} \quad$ Din-fen Zeng ${ }^{a} \quad$ Yu Qiu Zhou ${ }^{a}$ \\ Rong Hao Sun ${ }^{a}$ Chun Yan Shui ${ }^{a}$ Jiao Pei ${ }^{b}$ Wei Liu ${ }^{c}$ Xu Wang ${ }^{d}$ \\ Zheng Hua Jiang e Zheng Qi Tang ${ }^{f}$ Jian Jiang ${ }^{a}$ Wei Wang ${ }^{a}$ \\ aDepartment of Head and Neck Surgery, Sichuan Cancer Hospital and Institute, Sichuan \\ Cancer Center, School of Medicine, University of Electronic Science and Technology of \\ China, Chengdu, China; ${ }^{b}$ Department of GCP Center, Sichuan Cancer Hospital and Institute, \\ Sichuan Cancer Center, School of Medicine, University of Electronic Science and Technology \\ of China, Chengdu, China; ' Department of Otolaryngology Head and Neck Surgery, The \\ Affiliated Hospital of Southwest Medical University, Luzhou, China; ${ }^{d}$ Chengdu Medical \\ College, Chengdu, China; ${ }^{e}$ Department of Head and Neck Surgery, Central Hospital of \\ Mianyang City, Mianyang, China; ${ }^{\circ}$ Department of Head and Neck Surgery, The Third \\ People's Hospital of Zigong City, Zigong, China
}

\section{Keywords}

Tongue cancer - Reconstruction - Radial forearm free flap - Anterolateral thigh flap · Comparative analysis

\begin{abstract}
Background: Surgery is still the preferred treatment for tongue cancer. Reconstruction should be performed immediately after extensive resection of the tumor. The purpose of this study was to investigate the clinical effect, advantages, and disadvantages of radial forearm free (RFF) flap and anterolateral thigh (ALT) flap in tongue reconstruction after radical resection of tongue cancer. Methods: Thirty-nine cases of tongue reconstruction with RFF flap or ALT flap from 2014 to 2018 were analyzed. The survival of the flap, the functional status after repair, and the influence on the donor area were examined, in addition to the advantages and disadvantages of the flap and the critical points of the technique. Results: Twenty-one cases with RFF flaps and 18 cases with ALT flaps showed complete flap survival. Among them, 1 case in-
\end{abstract}

Y.-C.C. and Y.Q.Z. contributed equally to this work and served as co-first authors. C.L. and R.H.S. contributed equally to this work and served as co-corresponding authors. 
volved a venous vessel crisis after an ALT operation, and the flap survived after reoperation after thrombus removal and anastomosis. The recovery of tongue function was as follows: 41.0\% patients exhibited normal speech, $43.6 \%$ patients exhibited near-normal speech, $12.8 \%$ patients exhibited vague speech, and $2.6 \%$ patients could not speak. There was no significant difference between the 2 groups ( $p=0.134$ ). The recovery of tongue flexibility was as follows: $41.0 \%$ of the patients had normal postoperative tongue flexibility, $43.6 \%$ of the patients had slightly limited tongue flexibility, $12.8 \%$ of the patients had severely limited tongue flexibility, and $2.6 \%$ of the patients were completely limited. The difference between the 2 groups was statistically significant $(p=0.045)$. The postoperative diet of patients was as follows: $51.3 \%$ of patients had a regular diet, $33.3 \%$ of patients had soft foods, $12.8 \%$ of patients received a fluid diet, and $2.6 \%$ of patients could not eat after the operation. There was no significant difference between the 2 groups ( $p=0.209$ ). The satisfaction of donor area was as follows: $46.2 \%$ of the patients were satisfied with the donor area, $51.3 \%$ of the patients were basically satisfied with the donor area satisfaction, and $2.6 \%$ of the patients were not satisfied with the donor area satisfaction. There was no significant difference between the 2 groups $(p=0.809)$. Conclusion: The RFF flap is the most widely used technique in tongue reconstruction, especially in patients with tongue defects less than half of tongue tissue. However, for a large number of tissue defects caused by radical resection of advanced tongue cancer, the ALT flaps can provide a sufficient tissue volume, conceal scars after the operation, cause fewer complications in the donor area, and facilitate tongue function and aesthetic quality.

(c) 2019 S. Karger AG, Basel

\section{Introduction}

Tongue cancer is the most common malignant tumor of oral cancer, and the incidence of tongue cancer has increased in the past 30 years [1,2]. Surgical resection is an essential strategy for the treatment of tongue cancer. Radical resection can obtain a satisfactory 5-year survival rate $[3,4]$. However, the tongue is a critical organ in speaking, swallowing, and assisting the oral cavity to perform masticatory functions. Radical resection of tongue cancer often results in corresponding defects, which have a negative impact on the service and appearance of the tongue and a severe impact on the postoperative health-related quality of life in patients. Therefore, tongue reconstruction must be performed to maximize the recovery of tongue function and improve the patient's quality of life when tongue defect reaches a certain limit [5, 6]. However, there is no consensus on how to repair some tongue defects after radical surgery. At present, most scholars believe that different tissue flaps should be selected according to the defect size, location, and other soft and hard tissue defects [5, 7]. The purpose of this study is to examine the clinical effect, advantages, and disadvantages of the radial forearm free (RFF) and anterolateral thigh (ALT) flaps in tongue reconstruction after a tongue cancer surgery.

\section{Materials and Methods}

The research program was approved by the Ethics Committee of the Sichuan Cancer Hospital (SCCHEC2015016). This retrospective study included 39 patients with tongue reconstruction after tongue cancer resection with RFF (21 cases) and ALT (18 cases) from January 2014 to May 2018. Surgical and pathological records for each case were reviewed (Table 1). The type and size of the flap, speech, tongue flexibility, deglutition, and patient satisfaction with the donor appearance were evaluated half a year after operation. In speech function assessments, patients read a 200-word essay, and the accuracy of the text was rated as follows: (1) normal (accuracy over 90\%); (2) near-normal (accuracy over 75\%); (3) vague (accuracy $<50 \%$ ); 
Cai et al.: Comparative Analysis of RFF Flap and ALT Flap in Tongue Reconstruction after Radical Resection of Tongue Cancer

Table 1. Patient clinical data

\begin{tabular}{lrr}
\hline Clinical data & Examples $(n=39)$ & Percentage \\
\hline Gender & & \\
Female & 20 & 51.3 \\
Male & 19 & 48.7 \\
TNM staging & & \\
T1N0M0 & 2 & 5.2 \\
T2N0M0 & 17 & 43.6 \\
T2N1M0 & 1 & 2.6 \\
T2N2M0 & 3 & 7.7 \\
T3N0M0 & 7 & 17.9 \\
T4N0M0 & 4 & 10.3 \\
T4N1M0 & 1 & 2.6 \\
T4N2M0 & 4 & 10.3 \\
Pathological type & & 87.2 \\
SCC & 34 & 10.3 \\
ACC & 4 & 2.6 \\
Sarcomatoid carcinoma & 1 & 53.8 \\
Differentiation degree & & 20.5 \\
Well-differentiated & 8 & 12.8 \\
Moderately differentiated & 5 & 46.2 \\
Poorly differentiated & & 53.8 \\
Free flap & 21 & \\
ALT & & \\
RFF & & \\
\hline
\end{tabular}

RFF, radial forearm free; ALT, anterolateral thigh; SCC, squamous cell carcinoma; ACC, adenoid cystic carcinoma.

or (4) unable to speak. The flexibility of the tongue was graded as follows: (1) normal (can protrude from the mouth); (2) slightly limited (can protrude only to the lip); (3) severely limited (cannot reach the teeth); or (4) completely limited (fixed). Deglutition was subjectively estimated based on the diet feedback from patients and was classified follows: (1) regular diet; (2) soft diet; (3) fluid diet; or (4) nasal feeding. Donor satisfaction was classified as follows: (1) satisfied; (2) basically satisfied; and (3) not satisfied [7, 8] A surgeon performed the clinical evaluation.

All patients underwent radical resection of tongue cancer. The mandibular function and lingual gingival mucosa were preserved in the uninvaded early tongue carcinoma, while in the severe advanced tongue carcinoma, the median lip incision flap was turned over and mandibular alveolar resection was performed. Bilateral neck lymph node dissection was performed in patients with tumors extending across the midline, lymph node dissection in the I-III area was performed in patients with stage cN0, functional neck dissection was performed in patients with stage $\mathrm{cN} 1$, and radical neck dissection was performed in patients with lymph node invasion. The intraoperative frozen pathological examination ensured that the incisal margin was negative. At the same time, the flap was prepared by the recipient blood vessel. The branches of the external jugular vein, facial arteriovenous, and superior thyroid artery were preserved according to the specific conditions, and the thread was used for vascular anastomosis. Through the tunnel from the maxillofacial region to the neck, the vascular pedicle was passed through the tube to the submaxillary region. The artery was anastomosed first, and then the vein was anastomosed. After the vascular anastomosis was completed, the flap was sutured with the wound surface of the recipient area. Rubber drainage strips were placed in the anastomotic area, and the broad surface of the flap and drainage tubes were positioned in the neck.

RFF Harvesting

An Allen test was performed before the operation to avoid infusion into the donor forearm. The radial forearm flap centered on the cephalic vein was designed according to the defect size and repair needs. The 
Cai et al.: Comparative Analysis of RFF Flap and ALT Flap in Tongue Reconstruction after Radical Resection of Tongue Cancer
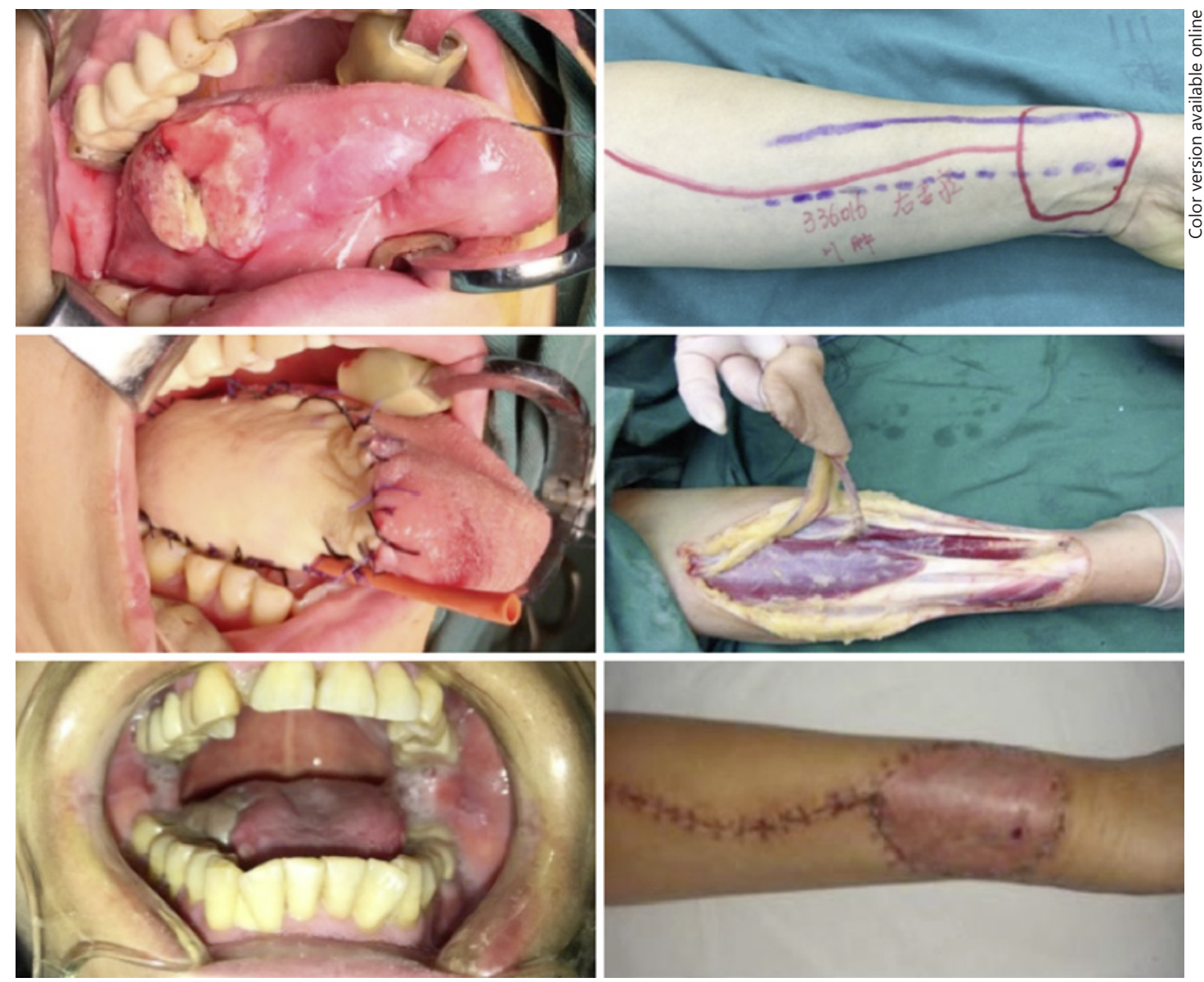

Fig. 1. Repair of postoperative defects from tongue carcinoma by using an RFF flap.

flap was cut along the outer edge of the flap to the superficial surface of the deep fascia and was lifted from distally to proximally on the surface of the myomembrane, and the cephalic vein was identified first. The subcutaneous connective tissue between the cephalic vein and the flap was preserved as much as possible to protect the communicating branches of the flap and the cephalic vein. The aponeurosis with a microvascular network on the surface of the flexor carpal flexor and the radial flexor were preserved completely to protect the flap and the radial artery. The perforating vessels between the veins were dissected in an anterograde direction, and the radial artery was dissected. The flap was gradually removed, and the radial artery and the cephalic vein were cut off and ligated at the distal end of the flap. In this procedure, the pedicle should be dissected to the cubital fossa, proper subcutaneous tissue should be preserved around the vein, the position of the pedicle should be determined according to the length of the vascular pedicle, the median nerve should not be damaged, and the pedicle of the flap should be cut off. The wound cavity was washed, and a negative pressure drainage tube was inserted. An abdominal skin graft was performed for a secondary defect of the forearm. The vessels of the recipient area were the facial artery, superior thyroid artery, external jugular vein, and facial vein (Fig. 1).

\section{ALT Harvesting}

The vessels of the perforating branch of the ultrasonic Doppler skin were located around the midpoint of the line from the anterior superior iliac ridge to the lateral patella before the operation. According to the size of the defect, the flap was designed with this point as the center. First, a medial incision and incisions of the skin and subcutaneous tissue were made. The perforating branch was dissected on the superficial facet of the deep fascia lata from inward and outward directions, the perforating branch was dissected retrogradely, the fascia between the rectus femoris and lateral femoris was opened, and the rectus femoris muscle 
Cai et al.: Comparative Analysis of RFF Flap and ALT Flap in Tongue Reconstruction after Radical Resection of Tongue Cancer
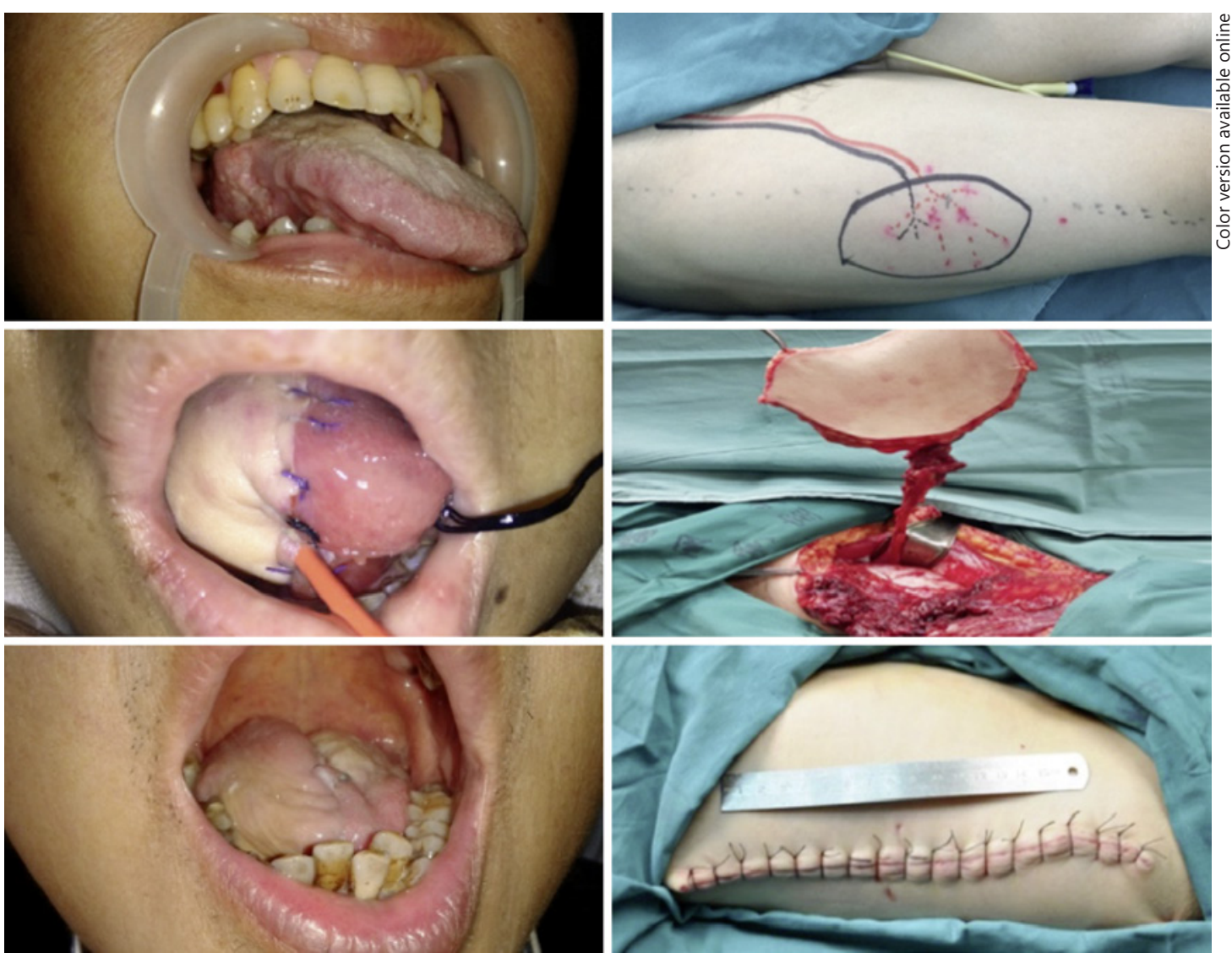

Fig. 2. Repair of postoperative defects from tongue carcinoma by using an ALT flap.

was opened to the medial side. The vascular and nerve bundles of the descending branch of the lateral femoral circumflex artery were exposed and confirmed as a descending branch of the lateral femoral circumflex artery. The shape and size of the flap were designed with the position of the perforating branch passing through the superficial surface of the muscle fascia as the axis, and the edge of the flap was cut to the superficial surface of the muscle fascia. The anatomy was examined along the perforating vessels from the distal to proximal ends to the descending branch of the lateral circumflex femoral artery. If the type of perforator was a cutaneous perforator, a few lateral femoral muscles on the surface of the vessel reached the descending branch of the lateral femoral artery. The proximal incision was prolonged, the lateral circumflex femoral artery was cut off, the descending branch of the vein was separated, and the main trunk was dissected to its beginning. When the recipient area was ready, the vascular pedicle could be ligated according to the length of the recipient area. The vessels of the recipient area were the facial artery, superior thyroid artery, external jugular vein, and facial vein. The donor areas were closed directly (Fig. 2).

Data analysis and statistics were conducted with the help of a professional statistician. All analyses were carried out using SPSS statistical software version 13.0 (SPSS, Inc., Chicago, IL, USA). The Kruskal Wallis test was used to analyze the difference between groups; $p<0.05$ was considered to indicate statistical significance.

\section{Results}

The study lasted 48 months and included 19 males (48.7\%) and 20 females (51.3\%), with an average age of 53.1 years (26-73 years). According to the (IUCC) TNM staging criteria of the International Commission on Oncology in 2002, there were 2 cases of stage T1N0M0, 17 
Cai et al.: Comparative Analysis of RFF Flap and ALT Flap in Tongue Reconstruction

Table 2. Patient and flap characteristics of RFF

\begin{tabular}{|c|c|c|c|c|c|c|c|c|c|c|c|c|}
\hline Number & Sex & $\begin{array}{l}\text { Age, } \\
\text { years }\end{array}$ & $\mathrm{T}$ class & $\begin{array}{l}\text { Pathological } \\
\text { type }\end{array}$ & $\begin{array}{l}\text { Tumor } \\
\text { size, cm }\end{array}$ & $\begin{array}{l}\text { Flap size, } \\
\mathrm{cm}\end{array}$ & $\begin{array}{l}\text { Operation } \\
\text { time, h }\end{array}$ & $\begin{array}{l}\text { Bleeding } \\
\text { volume, } \mathrm{mL}\end{array}$ & Speech & $\begin{array}{l}\text { Tongue } \\
\text { mobility }\end{array}$ & Dietary & $\begin{array}{l}\text { Donor } \\
\text { site }\end{array}$ \\
\hline 1 & M & 40 & $\mathrm{~T} 2$ & SCC & $2.0 \times 3.0$ & $6.0 \times 7.0$ & 6.2 & 1,000 & 1 & 1 & 1 & 2 \\
\hline 2 & M & 42 & $\mathrm{~T} 3$ & SCC & $2.8 \times 1.8$ & $5.0 \times 7.0$ & 6.4 & 1,000 & 1 & 1 & 1 & 2 \\
\hline 3 & $\mathrm{~F}$ & 43 & $\mathrm{~T} 4$ & SCC & $3.4 \times 2.1$ & $5.0 \times 7.0$ & 8.0 & 800 & 2 & 1 & 1 & 2 \\
\hline 4 & $\mathrm{~F}$ & 45 & $\mathrm{~T} 2$ & SCC & $4.2 \times 3.9$ & $7.0 \times 6.0$ & 7.0 & 500 & 2 & 2 & 2 & 2 \\
\hline 5 & $\mathrm{~F}$ & 48 & $\mathrm{~T} 3$ & SCC & $5.4 \times 3.0$ & $8.0 \times 6.0$ & 6.0 & 200 & 2 & 2 & 2 & 1 \\
\hline 6 & M & 49 & $\mathrm{~T} 2$ & SCC & $1.8 \times 1.0$ & $7.0 \times 5.0$ & 7.4 & 300 & 2 & 2 & 2 & 2 \\
\hline 7 & $\mathrm{~F}$ & 50 & $\mathrm{~T} 1$ & SCC & $1.6 \times 1.0$ & $5.0 \times 6.0$ & 5.0 & 100 & 2 & 2 & 3 & 1 \\
\hline 8 & M & 50 & $\mathrm{~T} 2$ & SCC & $2.9 \times 2.1$ & $7.0 \times 6.0$ & 7.15 & 1,000 & 1 & 2 & 2 & 2 \\
\hline 9 & M & 51 & T3 & SCC & $2.4 \times 2.6$ & $5.0 \times 7.0$ & 10.0 & 400 & 1 & 1 & 1 & 1 \\
\hline 10 & $\mathrm{~F}$ & 52 & $\mathrm{~T} 2$ & SCC & $1.7 \times 1.1$ & $5.0 \times 7.0$ & 5.3 & 100 & 1 & 1 & 1 & 1 \\
\hline 11 & M & 53 & $\mathrm{~T} 4$ & SCC & $2.7 \times 1.2$ & $6.0 \times 5.0$ & 10.0 & 150 & 2 & 2 & 1 & 1 \\
\hline 12 & $\mathrm{~F}$ & 53 & $\mathrm{~T} 3$ & SCC & $1.7 \times 1.0$ & $5.0 \times 7.0$ & 5.0 & 200 & 3 & 3 & 3 & 2 \\
\hline 13 & $\mathrm{~F}$ & 54 & $\mathrm{~T} 2$ & SCC & $3.0 \times 4.0$ & $6.0 \times 8.0$ & 6.4 & 1,000 & 1 & 1 & 1 & 2 \\
\hline 14 & $\mathrm{~F}$ & 55 & $\mathrm{~T} 2$ & SCC & $3.0 \times 2.0$ & $6.0 \times 7.0$ & 10.0 & 400 & 2 & 1 & 2 & 1 \\
\hline 15 & $\mathrm{~F}$ & 60 & $\mathrm{~T} 2$ & SCC & $1.0 \times 2.0$ & $5.0 \times 6.0$ & 10.0 & 800 & 2 & 1 & 1 & 2 \\
\hline 16 & $\mathrm{~F}$ & 61 & T3 & SCC & $1.4 \times 1.1$ & $5.0 \times 7.0$ & 7.5 & 800 & 1 & 1 & 1 & 1 \\
\hline 17 & $\mathrm{~F}$ & 62 & $\mathrm{~T} 2$ & SCC & $1.6 \times 1.4$ & $5.0 \times 7.0$ & 7.0 & 300 & 1 & 2 & 1 & 2 \\
\hline 18 & M & 62 & $\mathrm{~T} 2$ & SCC & $2.8 \times 2.4$ & $6.0 \times 7.0$ & 10.0 & 300 & 1 & 1 & 1 & 1 \\
\hline 19 & $\mathrm{~F}$ & 63 & $\mathrm{~T} 1$ & SCC & $2.5 \times 2.6$ & $6.0 \times 7.0$ & 7.0 & 300 & 2 & 2 & 1 & 2 \\
\hline 20 & $\mathrm{~F}$ & 64 & T3 & SCC & $2.1 \times 1.0$ & $5.0 \times 6.0$ & 6.45 & 200 & 2 & 2 & 3 & 2 \\
\hline 21 & $\mathrm{~F}$ & 66 & $\mathrm{~T} 2$ & SCC & $1.4 \times 1.1$ & $5.0 \times 7.0$ & 5.3 & 400 & 1 & 1 & 1 & 1 \\
\hline
\end{tabular}

RFF, radial forearm free; SCC, squamous cell carcinoma.

Table 3. Functional results of free flap reconstruction

\begin{tabular}{lllll}
\hline Characteristic & $\begin{array}{l}\text { Speech, } \\
\text { median } \pm \text { IQR }\end{array}$ & $\begin{array}{l}\text { Tongue mobility, } \\
\text { median } \pm \text { IQR }\end{array}$ & $\begin{array}{l}\text { Dietary, } \\
\text { median } \pm \text { IQR }\end{array}$ & $\begin{array}{l}\text { Satisfaction } \\
\text { of donor area, } \\
\text { median } \pm \text { IQR }\end{array}$ \\
\hline ALT & $2.0 \pm 1.0$ & $2.0 \pm 1.0$ & $1.0 \pm 1.0$ & $2.0 \pm 1.0$ \\
RFF & $2.0 \pm 1.0$ & $2.0 \pm 1.0$ & $1.0 \pm 1.0$ & $2.0 \pm 1.0$ \\
\hline$p$ value & 0.134 & 0.045 & 0.209 & 0.809 \\
\hline
\end{tabular}

IQR, interquartile range; RFF, radial forearm free; ALT, anterolateral thigh.

cases of stage T2N0M0, 1 case of stage T2N1M0, 3 cases of stage T2N2M0, 7 cases of stage T3N0M0, 4 cases of stage T4N0M0, 1 case of stage T4N1M0, and 4 cases of stage T4N2M0. There were 34 cases of squamous cell carcinoma, 4 cases of adenoid cystic carcinoma, and 1 case of sarcomatoid carcinoma. All the patients were followed up for 6-48 months. Twentyfive patients received postoperative adjuvant radiotherapy at a dose of 55-60 Gy, 2 of whom showed recurrent disease. The overall survival rate was $84.6 \%(33 / 39)$.

Twenty-one patients underwent tongue reconstruction with an RFF flap (Table 2). The mean operation time was $7.3 \mathrm{~h}$, the average bleeding volume was $488 \mathrm{~mL}$, and the flap measured $5.0 \times 6.0-6.0 \times 8.0 \mathrm{~cm}$. In these patients, 2 cases were repaired in the T1 stage, 11 cases in T2 stage, 6 cases in T3 stage, and 2 cases in the T4 stage. All the flaps survived; in 1 female with diabetes, necrosis was present in the tip (approximately 1/4) of the flap, and the flap survived after pruning the necrotic front end. Postoperative functional evaluation is detailed (Table 3). 


\begin{tabular}{l|l}
\hline ORL 2019;81:252-264 \\
\hline DOI: 10.1159/000502151 & $\begin{array}{l}\text { @ 2019 S. Karger AG, Basel } \\
\text { www.karger.com/orl }\end{array}$ \\
\hline
\end{tabular}

Cai et al.: Comparative Analysis of RFF Flap and ALT Flap in Tongue Reconstruction after Radical Resection of Tongue Cancer

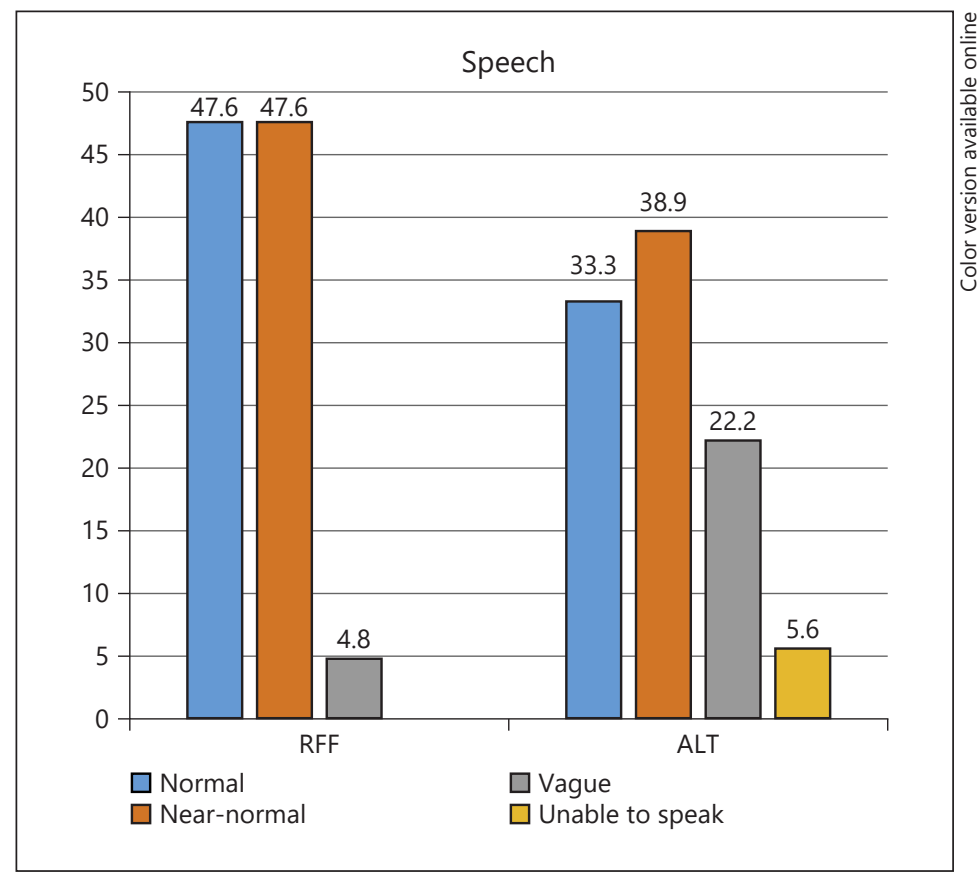

Fig. 3. Speech contrast between the 2 groups. RFF, radial forearm free; ALT, anterolateral thigh.

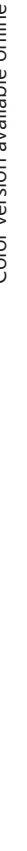

Table 4. Patient and flap characteristics of ALT

\begin{tabular}{|c|c|c|c|c|c|c|c|c|c|c|c|c|}
\hline Number & Gender & $\begin{array}{l}\text { Age, } \\
\text { years }\end{array}$ & T class & $\begin{array}{l}\text { Pathological } \\
\text { type }\end{array}$ & $\begin{array}{l}\text { Tumor size, } \\
\mathrm{cm}\end{array}$ & $\begin{array}{l}\text { Flap size, } \\
\mathrm{cm}\end{array}$ & $\begin{array}{l}\text { Operation } \\
\text { time, } \mathrm{h}\end{array}$ & $\begin{array}{l}\text { Bleeding } \\
\text { volume, } \mathrm{mL}\end{array}$ & Speech & $\begin{array}{l}\text { Tongue } \\
\text { mobility }\end{array}$ & Dietary & $\begin{array}{l}\text { Donor } \\
\text { site }\end{array}$ \\
\hline 1 & M & 26 & $\mathrm{~T} 2$ & ACC & $2.2 \times 1.3$ & $5.0 \times 6.0$ & 10.3 & 100 & 2 & 2 & 1 & 1 \\
\hline 2 & $\mathrm{~F}$ & 31 & $\mathrm{~T} 4$ & ACC & $3.8 \times 2.5$ & $6.0 \times 7.0$ & 6.3 & 300 & 2 & 1 & 1 & 2 \\
\hline 3 & M & 33 & $\mathrm{~T} 4$ & SCC & $3.6 \times 3.5$ & $6.0 \times 7.0$ & 9.0 & 1,200 & 3 & 3 & 3 & 2 \\
\hline 4 & M & 36 & $\mathrm{~T} 3$ & ACC & $1.6 \times 1.7$ & $5.0 \times 6.0$ & 9.0 & 500 & 1 & 2 & 1 & 1 \\
\hline 5 & $\mathrm{~F}$ & 42 & $\mathrm{~T} 4$ & $\begin{array}{l}\text { Sarcomatoid } \\
\text { carcinoma }\end{array}$ & $5.1 \times 4.7$ & $10.0 \times 14.0$ & 10.3 & 850 & 4 & 4 & 4 & 3 \\
\hline 6 & $\mathrm{~F}$ & 48 & $\mathrm{~T} 4$ & ACC & $2.3 \times 1.3$ & $5.0 \times 6.0$ & 14.0 & 800 & 2 & 3 & 2 & 1 \\
\hline 7 & M & 49 & $\mathrm{~T} 2$ & SCC & $1.0 \times 1.8$ & $6.0 \times 8.0$ & 7.0 & 300 & 2 & 2 & 2 & 1 \\
\hline 8 & $\mathrm{~F}$ & 51 & $\mathrm{~T} 2$ & SCC & $2.2 \times 1.0$ & $6.0 \times 5.0$ & 7.0 & 600 & 1 & 1 & 1 & 1 \\
\hline 9 & M & 52 & $\mathrm{~T} 4$ & SCC & $2.4 \times 1.0$ & $6.0 \times 8.0$ & 6.4 & 200 & 3 & 3 & 2 & 2 \\
\hline 10 & M & 54 & $\mathrm{~T} 2$ & SCC & $2.4 \times 1.0$ & $5.0 \times 6.0$ & 11.0 & 700 & 1 & 2 & 2 & 2 \\
\hline 11 & M & 59 & $\mathrm{~T} 2$ & SCC & $2.2 \times 2.0$ & $5.0 \times 6.0$ & 9.3 & 800 & 3 & 2 & 2 & 2 \\
\hline 12 & $\mathrm{~F}$ & 59 & $\mathrm{~T} 2$ & SCC & $3.5 \times 2.1$ & $5.0 \times 7.0$ & 6.4 & 400 & 1 & 1 & 1 & 1 \\
\hline 13 & M & 63 & $\mathrm{~T} 4$ & SCC & $1.8 \times 1.2$ & $5.0 \times 6.0$ & 8.0 & 500 & 3 & 3 & 3 & 2 \\
\hline 14 & M & 65 & $\mathrm{~T} 2$ & SCC & $2.7 \times 1.0$ & $6.0 \times 8.0$ & 6.4 & 200 & 1 & 1 & 1 & 1 \\
\hline 15 & $\mathrm{~F}$ & 68 & $\mathrm{~T} 2$ & SCC & $3.0 \times 2.8$ & $6.0 \times 7.0$ & 8.3 & 800 & 2 & 2 & 2 & 2 \\
\hline 16 & M & 69 & $\mathrm{~T} 2$ & SCC & $4.2 \times 3.9$ & $5.0 \times 7.0$ & 6.4 & 500 & 2 & 2 & 2 & 1 \\
\hline 17 & M & 70 & $\mathrm{~T} 4$ & SCC & $3.0 \times 2.8$ & $5.0 \times 7.0$ & 10.0 & 600 & 2 & 2 & 2 & 2 \\
\hline 18 & M & 73 & $\mathrm{~T} 2$ & SCC & $5.1 \times 4.0$ & $8.0 \times 6.0$ & 10.0 & 800 & 1 & 1 & 1 & 1 \\
\hline
\end{tabular}

(Tables 2, 4) Speech: (1) normal; (2) near-normal; (3) vague; (4) unable to speak. Tongue mobility: (1) flexible; (2) slight limitation; (3) severe limitation; (4) complete limitation. Dietary: (1) regular diet; (2) soft food; (3) liquid diet; (4) nasal feeding. Satisfaction of donor area: (1) satisfied; (2) basically satisfied; (3) not satisfied. ALT, anterolateral thigh; SCC, squamous cell carcinoma; ACC, adenoid cystic carcinoma. 
Fig. 4. Tongue mobility contrast between the 2 groups. RFF, radial forearm free; ALT, anterolateral thigh.
Cai et al.: Comparative Analysis of RFF Flap and ALT Flap in Tongue Reconstruction after Radical Resection of Tongue Cancer

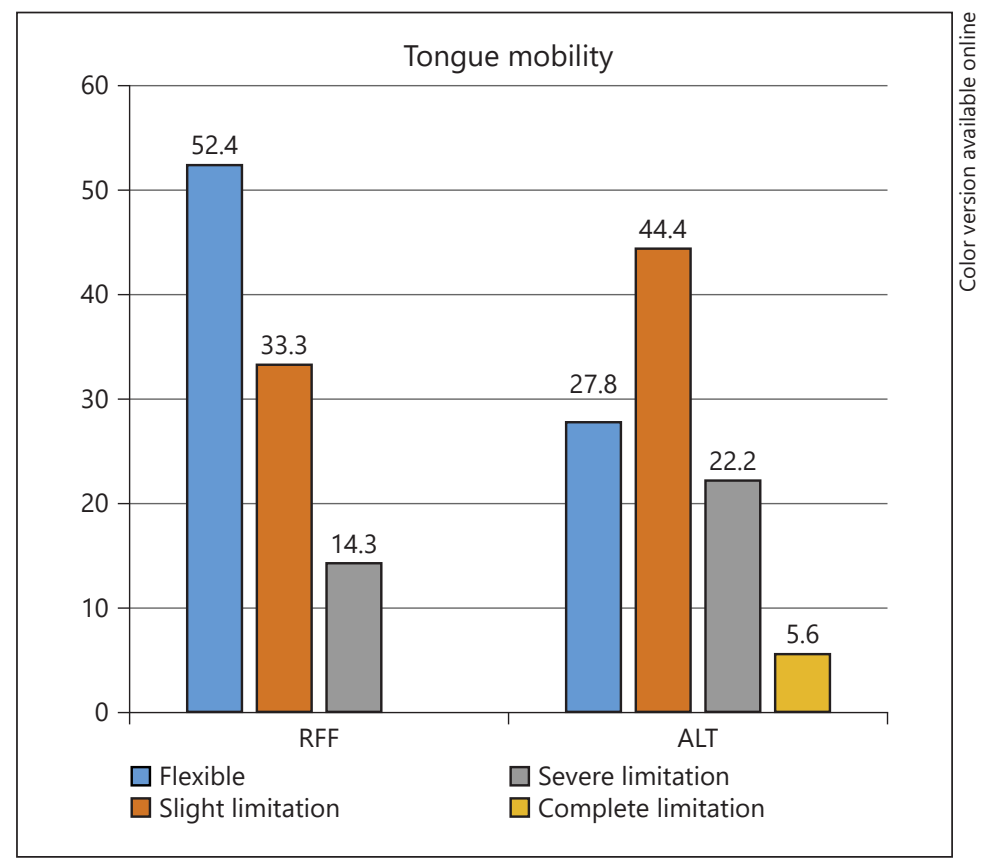

An ALT flap was used to reconstruct the tongue in 18 patients (Table 4). The mean operation time was $8.6 \mathrm{~h}$, the average blood loss was $563 \mathrm{~mL}$, and the skin flap measured $5.0 \times$ $6.0-10.0 \times 14.0 \mathrm{~cm}$. Among these patients, 10 cases were repaired in the T2 stage, 1 case in the T3 stage, and 7 cases in the T4 stage. All of the flaps survived, but 1 patient experienced a venous vascular crisis 3 days after the operation; the flap survived after reanastomosis of the thrombus. One patient presented fat liquefaction necrosis $(1.5 \times 2.0 \mathrm{~cm})$ in the suture area of the tongue tip and flap; the flap survived after debridement. A hematoma appeared in the donor area 5 days after debridement in 1 patient and healed normally after further debridement (Table 3).

A functional evaluation was conducted 6 months after the operation, $16(41.0 \%)$ patients had normal speech (10 in the RFF flap group, 6 in the ALT flap group), 17 (43.6\%) patients had near-normal speech (10 in the RFF flap group and 7 in the ALT flap group), 5 (12.8\%) patients had vague speech ( 1 in the RFF flap group, 4 in the ALT flap group), and 1 (2.6\%) patient was unable to speak (ALT flap group). It was apparent that the speech function of most of the patients recovered better after the operation. The RFF flap group showed better recovery than did the ALT flap group (Fig. 3). However, there was no significant difference between the 2 groups ( $p=0.134$ ).

There were $18(37.5 \%)$ patients with normal postoperative tongue flexibility, 21 (43.6\%) patients with slightly limited tongue flexibility, 8 (16.7\%) patients with severely limited tongue flexibility, and $1(2.1 \%)$ patient with completely limited tongue flexibility. The flexibility of the RFF flap group was better than that of the other 3 groups (Fig. 2), but there were no significant differences among the flap groups $(p=0.054)$. There were $16(41.0 \%)$ patients (11 in RFF flap group and 5 in the ALT flap group) with normal postoperative tongue flexibility, 17 (43.6\%; 9 in the RFF flap group and 8 in the ALT flap group) patients with slightly limited tongue flexibility, 5 (12.8\%) patients with severely limited tongue flexibility ( 1 in the RFF flap group and 4 in the ALT flap group), and 1 (2.6\%; ALT flap group) patient with completely limited tongue flexibility. It can be seen that the flexibility of tongue after RFF flap reconstruction was significantly better than that in the ALT flap group (Fig. 4). The difference between groups was statistically significant ( $p=0.045$ ). 
Fig. 5. Dietary contrast between the 2 groups. RFF, radial forearm free; ALT, anterolateral thigh.

Cai et al.: Comparative Analysis of RFF Flap and ALT Flap in Tongue Reconstruction after Radical Resection of Tongue Cancer

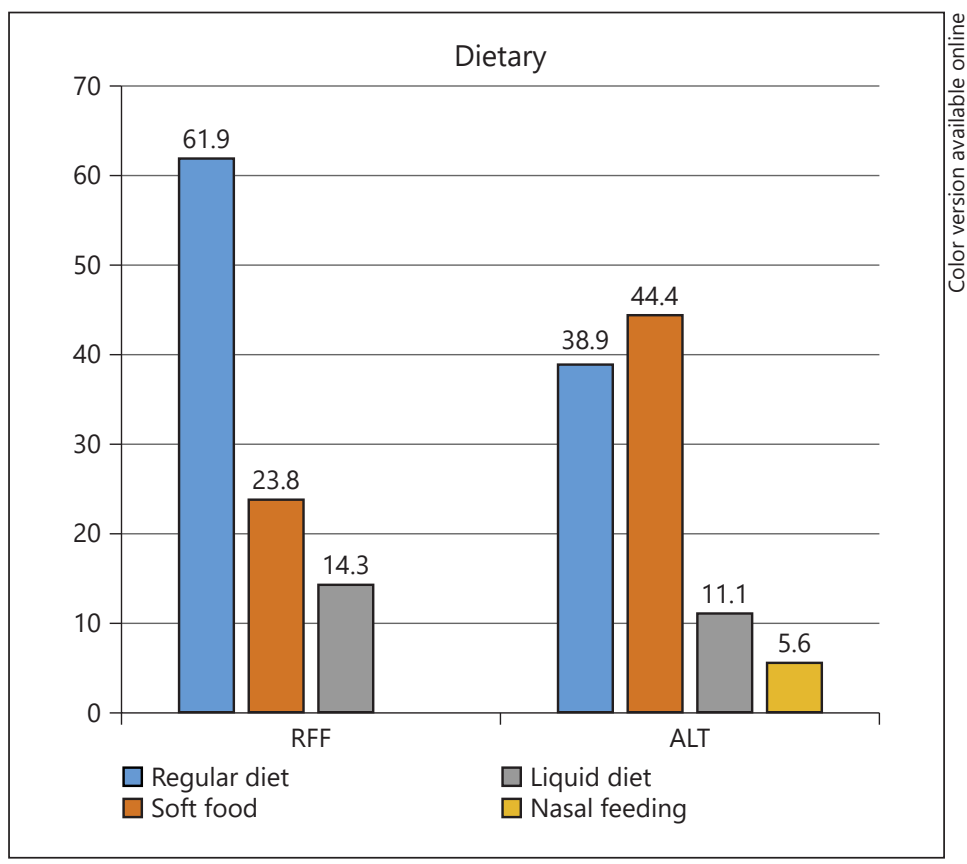

Deglutition (the deglutition was subjectively estimated based on the diet feedback from patients): Twenty (51.3\%; 13 in the RFF flap group and 7 in the ALT flap group) patients had a regular diet, $13(33.3 \%)$ patients had soft foods (5 in the RFF flap group and 8 in the ALT flap group), 5 (12.8\%) patients received a liquid diet (including 3 patients in the RFF flap group and 2 in the ALT flap group), and $1(2.6 \%)$ patient could not eat after operation (ALT flap group). Most of the patients had excellent recovery of feeding function after the surgery, and the RFF flap group showed better recover than did the ALT flap group (Fig. 5), but there was no significant difference between the 2 groups in this regard $(p=0.209)$. Eighteen patients were satisfied (46.2\%; 9 in the RFF flap group and 9 in the ALT flap group), 20 patients were basically satisfied (51.3\%; 12 in the RFF flap group and 8 in the ALT flap group), and 1 (2.6\%) patient was dissatisfied after operation (ALT flap group); there was no significant difference between the 2 groups ( $p=0.809$; Table 3 ).

\section{Discussion}

The tongue is an organ with complex physiological and social functions that are severely affected by tissue defects after the radical resection of tongue cancer. Partial tongue resection has a significant effect on quality of life. The reconstruction of tongue defects is very demanding, but their purpose is to reconstruct the appearance, eliminate dead space, and maintain the patient's ability to chew, swallow, and speak $[6,9]$. Pedicled flaps and free flaps are commonly used to repair tongue defects. However, some studies suggest that with the use of pedicle flaps, the mobility of the reconstructed tongue and the recovery of speech are affected by gravity causing tongue and pedicle drooping [10]. It is also suggested that reconstruction of the flap may impair the function of the residual tongue tissue due to the introduction of nonmotile tissue [11]. However, consecutive studies have shown that to improve postoperative speech and swallowing functions and the postoperative quality of life, free flap reconstruction is necessary for restoration of the tongue volume and better functional outcomes in patients who have undergone resection of more than half of the tongue volume [7, 12-14]. 
Fig. 6. Satisfaction of donor area contrast between the 2 groups. $\mathrm{RFF}$, radial forearm free; ALT, anterolateral thigh.

Cai et al.: Comparative Analysis of RFF Flap and ALT Flap in Tongue Reconstruction after Radical Resection of Tongue Cancer

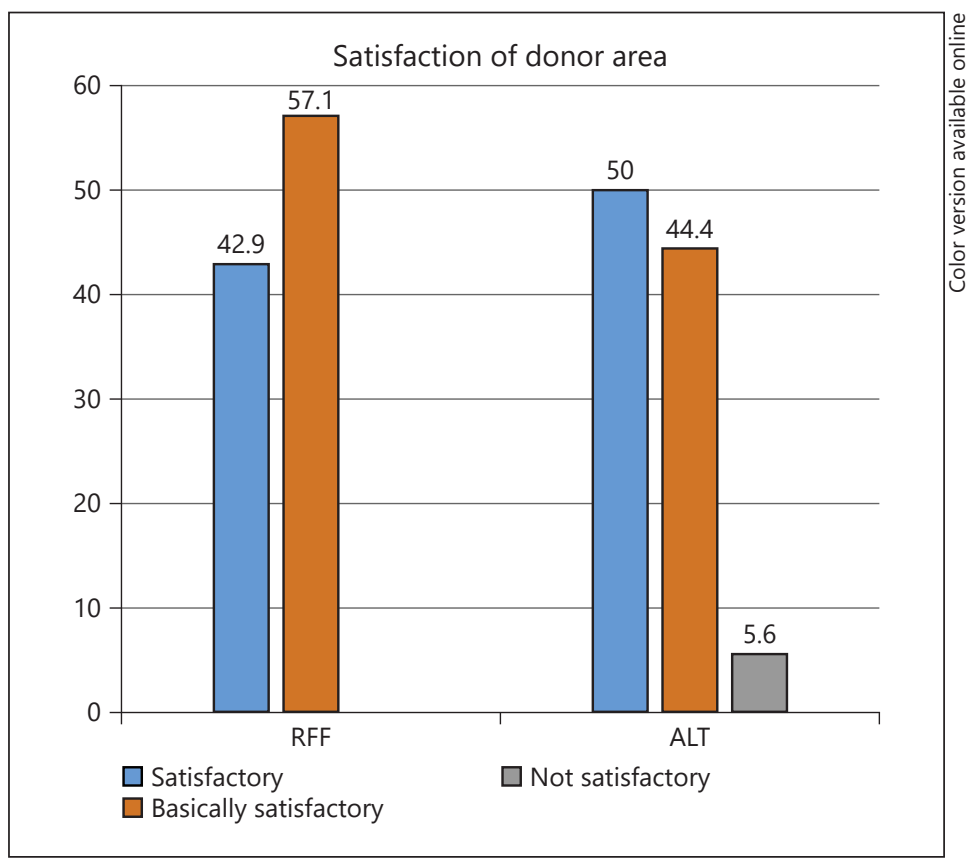

With the development of microsurgery, all kinds of free flaps have been used in tongue reconstruction. At present, RFF flaps [15, 16] and ALT flaps [13, 17] are widely used. Lateral upper arm free flaps $[8,18]$, perforator flaps from the lower leg [19], and deep inferior epigastric artery perforator flaps [20] have also been used in tongue reconstruction. However, it is not clear whether free flap reconstruction can improve quality of life, and the choice of the type of free flap reconstruction is still controversial.

The RFF flap is still the main flap currently used for tongue reconstruction [11]. In this study, 21 (63.8\%) patients were treated with an RFF flap for tongue reconstruction, which was also the main repair method. Postoperative functional evaluations revealed that after the operation, $47.6 \%$ of the patients had normal speech, $47.6 \%$ of the patients had near-normal speech, $61.9 \%$ of the patients ingested regular diet, and $23.8 \%$ of the patients received soft foods after surgery, which was better than that of the ALT flap group (Fig. 3, 5). The functional recovery of patients with tongue reconstruction with RFF flap was satisfactory, especially when the tongue defect involves 1/3-1/2 of the organ; 13 (61.9\%) patients of this study underwent repair of stage T1 and T2 defects. The postoperative tongue flexibility of the T2 defect in the RFF flap group was better than that of the ALT flap group ( $p=0.045$; Fig. 4). Thus, our study shows the RFF flap is the ideal choice for early stage tumors, the advantages of the RFF flap to be the following: (1) the diameter of the vessel is thicker, the pedicle of the vessel is longer, the diameter of the vessel is matched with the blood vessel of the neck, the flap is easy to anastomose, and the success rate of the flap is high; (2) the flap is thin and easy to shape, can be folded and twisted, and forms an ideal maxillary tongue groove; (3) furthermore, the RFF flap can be used in oral repair without the occurrence of bloating and has little effect on respiration and language after the operation. The main disadvantage of the RFF flap is that interception of the RFF involves cutting off the radial artery, which is the main artery for the forearm. This procedure can affect the sensation and motor function of the hand, and the concealment of the surgical wound is not ideal. Second, grafts are needed to block the donor area, and the scar is so apparent that it is difficult for many people to accept [21,22]. In this study, only 9 (42.9\%) patients were satisfied with the donor area postoperatively, which was lower than that of the ALT flap group (Fig. 6). 
Cai et al.: Comparative Analysis of RFF Flap and ALT Flap in Tongue Reconstruction after Radical Resection of Tongue Cancer

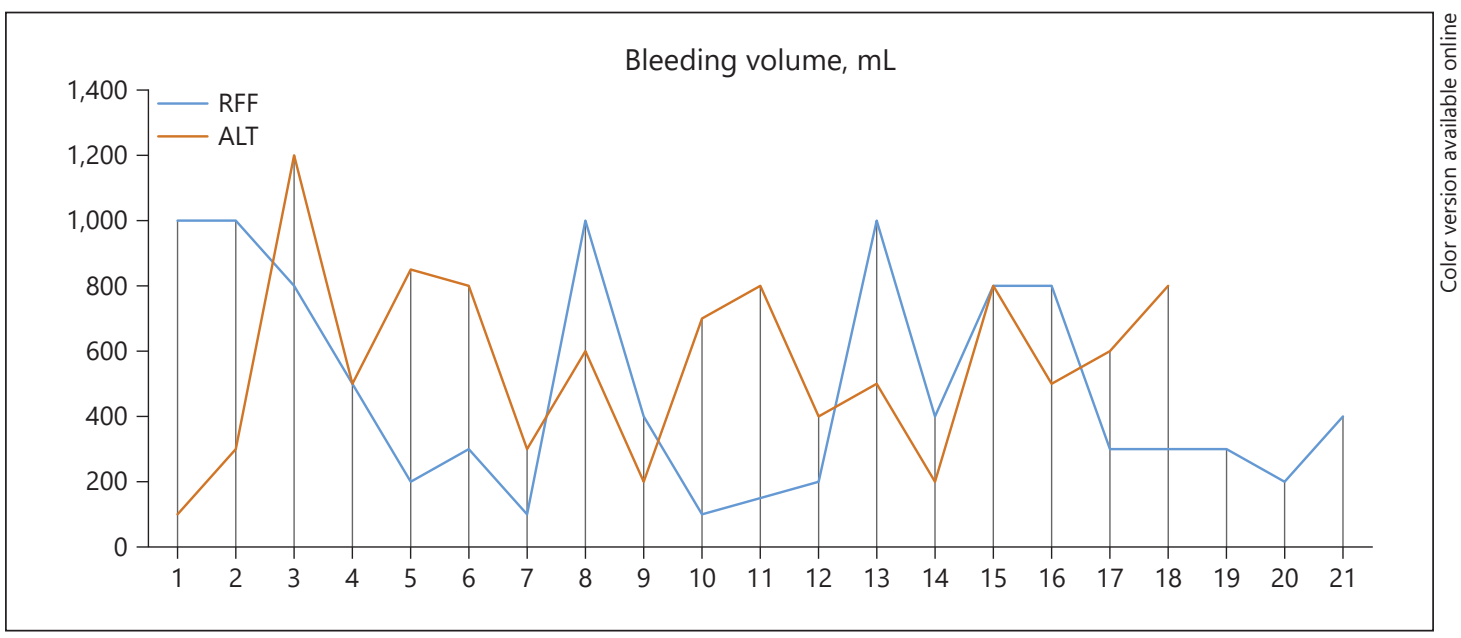

Fig. 7. Curve of blood volume between the 2 groups. RFF, radial forearm free; ALT, anterolateral thigh.

It is precisely because of some shortcomings of the RFF flap procedure that the ALT flap is widely used in tongue reconstruction, especially for a large number of tissue defects caused by the radical resection of advanced tongue cancer [13, 23], the ALT flap can provide a sufficient tissue volume [24]. In this study, 18 (37.5\%) patients received an ALT flap for tongue reconstruction. The postoperative functional evaluations revealed that $33.3 \%$ of the patients exhibited normal speech, $38.9 \%$ of the patients exhibited near-normal speech, and $22.2 \%$ of the patients exhibited vague speech after the operation. Furthermore, 38.9\% of the patients had a regular diet, $44.4 \%$ had a soft diet, and $11.1 \%$ received a liquid diet. These percentages were slightly lower than those after the RFF procedure. We conclude that most of the patients treated with ALT flaps have advanced tongue cancer, that the defect after the operation is more significant, that the repair flap is thicker, and that the duration of flap atrophy is relatively long. In this study, 8 (44. 4\%) patients received ALT flaps to repair T3 and T4 defects, which was one of the reasons why the operation time and the amount of intraoperative bleeding in the ALT flap group were greater than those in the RFF flap group (Fig. 7). An HRQOL study reported that the postoperative assessment time should be 12 months later as a critical factor [25]. In this study, the tongue function was evaluated 6 months after the operation. Fifty-five percent of the patients were satisfied, and $44.4 \%$ of the patients were basically satisfied with the donor area, slightly higher percentages than those for the RFF flap (Fig. 6). Our study shows that for patients with advanced tongue cancer, in which the tongue body defect is subtotal glossectomy or even total glossectomy involving resection of most of the tongue body and tongue root, the ALT flap is a better choice [26]. We detail what we think the advantages of the ALT flap in repairing the defects of tongue cancer after surgery below. (1) As a large area can be removed, sufficient tissue can be provided to fill the defect in the tongue root and mouth floor, and a sufficient quantity of tongue root tissue is the key to restoring the function of swallowing. (2) The length of vascular pedicle is up to $10-12 \mathrm{~cm}$, and the diameter of the vessel is large (approximately $2.5 \mathrm{~mm}$ ); in addition, the vessel is similar to that of the recipient area of the maxillofacial neck and is easy to anastomose. At the same time, there are 2 veins with a deep venous system, no valves, good reflux, and usually one anastomosis after tongue cancer. If 2 veins are anastomosed, the risk of vascular crisis is lower. (3) The main vessels of the limbs are not sacrificed, and there is a reduced incidence of donor area dysfunction and complications, which is easily accepted by young people. (4) The flap can carry the anterolateral femoral cutaneous nerve when necessary to produce a sensory flap. (5) The flap donor area is $<10 \mathrm{~cm}$ 
Cai et al.: Comparative Analysis of RFF Flap and ALT Flap in Tongue Reconstruction after Radical Resection of Tongue Cancer

in width and can be closed and sutured directly, without the need for skin grafting and without obvious effects on shape and function. (6) The posture is convenient, especially for head and neck surgery, and the donor area can be operated on at the same time, shortening the surgical time [27]. One shortcoming of the ALT flap is that the flap is perforating vessel, and the perforating branch is changeable, so the routine preoperative ultrasound Doppler skin perforator vessel localization is usually performed. There is also 3-dimensional reconstruction via CT/ MRI angiography to provide further intuitionistic information on the flow of blood vessels, branches, and blood flow axis [28].

\section{Conclusion}

In this study, an RFF flap was most commonly used in tongue reconstruction, especially in patients with tongue defect less than half of tongue tissue However, for a large number of tissue defects caused by radical resection of advanced tongue cancer, ALT flaps can provide a sufficient tissue volume, a concealed scar, and fewer complications in the donor area, both functional and aesthetic. Therefore, RFF flap is the ideal choice for early-stage tumors and the ALT flap is the flap of choice for large resections including the root of the tongue head, and neck surgeons should consider the biological behavior of the tumor, the general and local status of the patient, the history of treatment, the complications of the recipient area, and the function and appearance of the reconstruction, along with economic factors and the technical level of the operator before selecting the reconstruction strategy. An individualized functional repair strategy can thereby be realized after the completion of tongue cancer surgery.

\section{Acknowledgments}

This work was supported by the Major Science and Technology Project Foundation of Sichuan Science and Technology Department (19 YFS0337) and the Head and Neck Cancer Prevention and Treatment Project of Sichuan Province supported by the Scientific and Technological Innovation Team (2014TD0011).

\section{Disclosure Statement}

We have no conflicts of interest.

\section{Author Contributions}

C.L. is the corresponding author, and he contributed to the conception of the study. Y.-C.C. is the first author, and he contributed significantly to analysis and manuscript preparation. W.W., Y.Q.Z. and W.L. performed the data collection, while C.Y.S. performed the clinical evaluation. J.P. performed the data analyses; all authors read and approved the final manuscript.

\section{References}

1 Tota JE, Anderson WF, Coffey C, Califano J, Cozen W, Ferris RL, et al. Rising incidence of oral tongue cancer among white men and women in the United States, 1973-2012. Oral Oncol. 2017 Apr;67:146-52.

2 Krishnatreya M, Nandy P, Rahman T, Sharma JD, Das A, Kataki AC, et al. Characteristics of oral tongue and base of the tongue cancer: a hospital cancer registry based analysis. Asian Pac J Cancer Prev. 2015;16(4):1371-4.

3 Yu GP, Mehta V, Branovan D, Huang Q, Hashibe M, Zhang ZF, et al. Improved survival among patients with base of tongue and tonsil cancer in the United States. Cancer Causes Control. 2012 Jan;23(1):153-64.

4 Zhang L, Zhou X, Yao X, Wu Y, Zhang Q, Zhang L. Oral tongue cancer patients show a better overall survival than base of tongue cancer patients. J Cancer Res Clin Oncol. 2012 Feb;138(2):341-6. 
Cai et al.: Comparative Analysis of RFF Flap and ALT Flap in Tongue Reconstruction after Radical Resection of Tongue Cancer

5 Baas M, Duraku LS, Corten EM, Mureau MA. A systematic review on the sensory reinnervation of free flaps for tongue reconstruction: does improved sensibility imply functional benefits?. J Plast Reconstr Aesthet Surg. 2015 Aug;68(8):1025-35.

6 Khan MN, Perez E, Goljo E, Iloreta A, Park RC, Genden EM, et al. The price of free tissue transfer after tongue reconstruction: quantifying the risks. Laryngoscope. 2017 Jul;127(7):1551-7.

7 Ji YB, Cho YH, Song CM, Kim YH, Kim JT, Ahn HC, et al. Long-term functional outcomes after resection of tongue cancer: determining the optimal reconstruction method. Eur Arch Otorhinolaryngol. 2017 Oct;274(10): 3751-6.

8 Yang XD, Zhao SF, Wang YX, Li W, Zhang Q, Hong XW, et al. Use of Extended Lateral Upper Arm Free Flap for Tongue Reconstruction After Radical Glossectomy for Tongue Cancer. Aesthetic Plast Surg. 2015 Aug;39(4): 562-9.

9 Koshima I, Hosoda M, Moriguchi T, Kawada S. New multilobe "accordion" flaps for three-dimensional reconstruction of wide, full-thickness defects in the oral floor. Ann Plast Surg. 2000 Aug;45(2):187-92.

10 Liu J, Wu H, Zhu Z, Wu X, Tan H, Wang K. [Free anterolateral thigh myocutaneous flap for reconstruction of soft tissue defects following en block resection of tongue cancer]. Zhongguo Xiu Fu Chong Jian Wai Ke Za Zhi. 2010 Jan;24(1):82-6.

11 Hartl DM, Dauchy S, Escande C, Bretagne E, Janot F, Kolb F. Quality of life after free-flap tongue reconstruction. J Laryngol Otol. 2009 May;123(5):550-4.

12 Bokhari WA, Wang SJ. Tongue reconstruction: recent advances [J]. Curr Opin Otolaryngol Head Neck Surg. 2007 Aug;15(4):202-7.

13 Yu P. Reinnervated anterolateral thigh flap for tongue reconstruction. Head Neck. 2004 Dec;26(12):1038-44.

14 Yu P, Robb GL. Reconstruction for total and near-total glos- sectomy defects. Clin Plast Surg. 2005 Jul;32(3): 411-9.

15 Paydarfar JA, Patel UA. Submental Island Pedicled Flap vs Radial Forearm Free Flap for Oral Reconstruction. Arch Otolaryngol Head Neck Surg. 2011 Jan;137(1):82-7.

16 Seikaly H, Rieger J, Wolfaardt J, Moysa G, Harris J, Jha N. Functional outcomes after primary oropharyngeal cancer resection and reconstruction with the radial forearm free flap. Laryngoscope. 2003 May;113(5):897904.

17 Tong XJ, Tang ZG, Shan ZF, Guo XC. The anterolateral thigh flap for soft tissue reconstruction in patients with tongue squamous cell carcinoma. World J Surg Oncol. 2016 Aug;14(1):213.

18 Li C, Cai YC, Wang W, He YX, Lan XJ, Li QL, et al. [The role definition of lateral arm free flap in reconstruction after head and neck cancer surgery]. Zhonghua Er Bi Yan Hou Tou Jing Wai Ke Za Zhi. 2016 Feb;51(2):156-60.

19 Wolff KD, Rau A, Kolk A. Perforator flaps from the lower leg for intraoral reconstruction: experience of 131 flaps. J Craniomaxillofac Surg. 2018 Feb;46(2):338-45.

20 Masià J, Sommario M, Cervelli D, Vega C, León X, Pons G. Extended deep inferior epigastric artery perforator flap for head and neck reconstruction: a clinical experience with 100 patients. Head Neck. 2011 Sep;33(9): 1328-34.

21 Orlik JR, Horwich P, Bartlett C, Trites J, Hart R, Taylor SM. Long-term functional donor site morbidity of the free radial forearm flap in head and neck cancer survivors. J Otolaryngol Head Neck Surg. 2014 Jan;43(1):1.

22 Avery CM. Review of the radial free flap: is it still evolving, or is it facing extinction? Part one: soft-tissue radial flap. Br J Oral Maxillofac Surg. 2010 Jun;48(4):245-52.

23 Ali RS, Bluebond-Langner R, Rodriguez ED, Cheng MH. The versatility of the anterolateral thigh flap. Plast Reconstr Surg. 2009 Dec;124(6 Suppl):e395-407.

24 Zhang PP, Meng L, Shen J, Liu H, Zhang J, Xiang X, et al. Free radial forearm flap and anterolateral thigh flap for reconstruction of hemiglossectomy defects: A comparison of quality of life. J Craniomaxillofac Surg. 2018 Dec; 46(12):2157-63.

25 Ronis DL, Duffy SA, Fowler KE, Khan MJ, Terrell JE. Changes in quality of life over 1 year in patients with head and neck cancer. Arch Otolaryngol Head Neck Surg. 2008 Mar;134(3):241-8.

26 Chen H, Zhou N, Huang X, Song S. Comparison of morbidity after reconstruction of tongue defects with an anterolateral thigh cutaneous flap compared with a radial forearm free-flap: a meta-analysis. Br J Oral Maxillofac Surg. 2016 Dec;54(10):1095-101.

27 Deng TH, Li C, Cai YC. Advances in the application of anterolateral femoral perforator flap in the surgical application of head and neck tumors. Journal of Cancer Control and Treatment. 2016;29(2):97-101.

28 Tang T, Zhou P, Wang Z, Li C, Chen J, Cai Y, et al. [Application of CT angiography in design of anterolateral thigh perforator flap for reconstruction of defect after head and neck cancer resection]. Zhonghua Er Bi Yan Hou Tou Jing Wai Ke Za Zhi. 2015 May;50(5):383-7. 